\title{
Predicting Kidney Graft Survival Using Machine Learning Methods: Prediction Model Development and Feature Significance Analysis Study
}

Syed Asil Ali Naqvi ${ }^{1}$, MCS; Karthik Tennankore ${ }^{2}$, MD; Amanda Vinson ${ }^{2}$, MD; Patrice C Roy ${ }^{1}$ PhD; Syed Sibte Raza Abidi ${ }^{1}, \mathrm{PhD}$

${ }^{1}$ Department of Computer Science, Dalhousie University, Halifax, NS, Canada

${ }^{2}$ Division of Nephrology, Dalhousie University, Halifax, NS, Canada

\section{Corresponding Author:}

Syed Asil Ali Naqvi, MCS

Department of Computer Science

Dalhousie University

Goldberg Computer Science Bldg

6050 University Ave

Halifax, NS, B3H 1W5

Canada

Phone: 19023290504

Email: a.naqvi@dal.ca

\section{Abstract}

Background: Kidney transplantation is the optimal treatment for patients with end-stage renal disease. Short- and long-term kidney graft survival is influenced by a number of donor and recipient factors. Predicting the success of kidney transplantation is important for optimizing kidney allocation.

Objective: The aim of this study was to predict the risk of kidney graft failure across three temporal cohorts (within 1 year, within 5 years, and after 5 years following a transplant) based on donor and recipient characteristics. We analyzed a large data set comprising over 50,000 kidney transplants covering an approximate 20-year period.

Methods: We applied machine learning-based classification algorithms to develop prediction models for the risk of graft failure for three different temporal cohorts. Deep learning-based autoencoders were applied for data dimensionality reduction, which improved the prediction performance. The influence of features on graft survival for each cohort was studied by investigating a new nonoverlapping patient stratification approach.

Results: Our models predicted graft survival with area under the curve scores of $82 \%$ within 1 year, $69 \%$ within 5 years, and $81 \%$ within 17 years. The feature importance analysis elucidated the varying influence of clinical features on graft survival across the three different temporal cohorts.

Conclusions: In this study, we applied machine learning to develop risk prediction models for graft failure that demonstrated a high level of prediction performance. Acknowledging that these models performed better than those reported in the literature for existing risk prediction tools, future studies will focus on how best to incorporate these prediction models into clinical care algorithms to optimize the long-term health of kidney recipients.

(J Med Internet Res 2021;23(8):e26843) doi: 10.2196/26843

\section{KEYWORDS}

kidney transplantation; machine learning; predictive modeling; survival prediction; dimensionality reduction; feature sensitivity analysis 


\section{Introduction}

\section{Background}

Kidneys are vital for the health of an individual, as they filter waste products from the blood and produce hormones and urine [1]. Patients are considered to have end-stage renal disease when their kidney function falls below a specific threshold [2]. A lack of timely measures to prevent kidney failure results in premature death $[3,4]$.

Kidney transplantation [5,6] and dialysis are the two main treatments for kidney failure [7]. Kidney transplantation offers a survival advantage compared with other forms of kidney replacement therapy; however, the rate of graft loss following transplant is still undesirably high [8]. Kidneys are a limited resource, and optimizing the match between donors and recipients is crucial for improving outcomes after transplantation. Kidney transplant allocation is, in part, based on a number of donor-recipient-related factors that influence graft survival. Various clinical studies have been conducted on the influence of these factors on graft survival; however, given the complex interactions between these factors, there remains much to be learned in this area. Existing risk prediction models only have a limited ability to predict outcomes for kidney transplant recipients with receiver operating characteristic scores of 0.6-0.7 [9-11].

Prediction modeling using machine learning (ML) algorithms has gained attention in recent years [12] for predicting the success of clinical or surgical procedures (such as kidney transplant). ML algorithms autonomously learn the underlying associations between preprocedure clinical features and postprocedural outcomes to predict the outcome of the procedure for a given clinical case. In kidney transplant, ML-based prediction models, based on donor-recipient information, autonomously learn the underlying relationships between donor and recipient factors to predict transplant outcomes. Multiple studies have been conducted using ML methods to predict the kidney graft outcome [13-16]; however, the standard approach in nearly all the reviewed studies has been to select one or more arbitrary period starting from the date of transplant and applying classification algorithms for prediction. There is a clear need for further exploration of data stratification approaches and other ML methods with respect to feature engineering and prediction modeling.

\section{Objectives}

In this study, the intent is to investigate kidney transplant allograft survival, that is, estimating the time-to-event and the evolving influence of clinical features leading to an event-within three temporal cohorts of 1 year, $>1-5$ years, and $>5$ years of a kidney transplant. We predicted the outcome of graft failure after kidney transplant based on the analysis of donor and recipient features. We applied ML methods to (1) predict the graft status over different temporal periods and (2) analyze the changing effect of donor-recipient-related predictors across different periods. To develop the prediction models, we analyzed a large data set of over 50,000 transplants covering approximately a 20-year period of kidney transplants in the United States. To generate the clinically meaningful temporal cohorts, we experimented with two patient stratification approaches: (1) a novel nonoverlapping patient stratification approach, whereby a patient's graft failure was recorded only in the temporal cohort when it actually happened, that is, a graft failure event in the preceding cohort was not included and (2) the traditional overlapping patient stratification approach that provides an accumulative count of graft outcomes until a specific time point. To develop the prediction models, we investigated multiple ML algorithms using both patient stratification approaches. Nonoverlapping temporal cohorts were considered to investigate the influence of clinical features on predicting graft survival over time, as the temporal partitioning of the data allowed for the establishment of feature influence across distinct temporal windows. We applied the feature importance method based on the mean decrease in impurity (Gini).

The contributions of this research are as follows: (1) ML-based prediction models that are trained on a large data set, offering improved prediction performance compared with previous studies (previous graft prediction studies are based on a smaller number of transplants over a shorter period); (2) data dimensionality reduction based on a deep learning framework to handle the high-dimensional and complex kidney transplant data set; (3) a novel nonoverlapping patient stratification approach to provide fine-grained feature importance within a specific period while avoiding bias from preceding cohorts; (4) explaining the influence of the different clinical features, during different periods, toward the prediction performance of ML prediction models. This finding allows the selection of the most important features to predict graft outcomes within a specific temporal window; and (5) a comparison between the two stratification approaches with respect to the performance of the prediction models. The future practical outcome of this study is the provision of a data-driven decision support tool to assist nephrologists in the kidney allocation process by identifying the best donor and recipient pair that will lead to the highest likelihood of graft survival for a given recipient.

\section{Prior Work}

Patients can receive a kidney from either deceased donors or living donors. The donor-recipient matching process becomes relatively more complex with deceased donors because of the need to account for additional clinical factors (ie, prolonged cold ischemia time, prolonged wait times, and generally lower quality organs) [17]. Given the fact that kidney organs are a limited resource, it is important to have an efficient and effective donor-recipient matching process to ensure long-term graft survival [18].

Data-driven methods are now being used for organ matching; these methods are used to establish clinical compatibility beyond the blood group and tissue type. Conventional data-driven prediction methods use statistical techniques such as Cox proportional hazard models and Kaplan-Meier estimates to perform time-to-event analyses [19]. Significant research has been conducted with Cox-based models in the survival analysis of different organ transplants; however, these methods eventually lose predictive accuracy as the feature space increases $[13,14]$. 
ML-based data analysis to develop prediction models for predicting outcomes is usually performed using classification methods, whereas regression methods are used for time-to-event analysis. There are two prominent approaches to predict kidney allograft outcomes using ML-based classification methods. The first approach is to predict graft survival over time by dividing a longitudinal data set into different time cohorts based on the occurrence of a given adverse event or the last follow-up date from the date of transplant. Each time cohort has a binary target variable, that is, success or failure of the graft, which is used to train the classification model to predict graft survival $[15,16]$. The second approach is to predict the risk acuity associated with a graft within a period. Topuz et al [15] used this approach and divided the data set into three graft failure risk groups (high, medium, and low) across three different periods to predict the risk of graft failure within a specific time using classification methods. Li et al [20] used Bayes net to classify graft risk levels and predict graft survival.

Due to the high dimensionality of existing data sets for organ transplantation, feature selection is applied to filter out redundant features. A stacked autoencoder, which is an unsupervised neural network, is an efficient dimensionality reduction technique with promising performance for deep representation of medical data [21] that reconstructs its own inputs by first encoding them to a smaller size and then decoding back to the original inputs. A comparative study by Sadati et al [22] highlighted the efficacy of different types of autoencoders for data sets based on electronic health records.

Right-censored data are a common problem for survival analysis, as it represents cases for which the adverse event is not available or recorded because of either the subject having been lost to follow-up or not experiencing the event during the study period. Multiple approaches have been adopted in previous studies to address this problem. The study on kidney transplants by Topuz et al [15] discarded all the right-censored data before 7 years from the time of transplant and included the remaining transplants that took place after that time point in the low-risk group. In a study predicting heart transplant outcomes, the data set was divided into three different time cohorts $(1,5$, and 9 years) to predict the status of the graft. Patients who did not have graft failure during a particular time cohort were censored, and all the patients beyond that time cohort were considered to have successful transplants [23].
The influence of clinical features (or clinical predictors) on graft survival tends to vary over time [16], as shown using a heat map [24]. Dag et al [23] analyzed the changing significance of features for three overlapping time cohorts $(1,5$, and 9 years). They deduced that certain types of features perform well in the long term compared with the short and medium terms. For instance, socioeconomic factors were more influential in their 9 -year time cohort as they covered major variations in the data. It is important to note here that feature significance over time can only be substantiated if the analysis is performed with nonoverlapping cohorts to avoid any bias introduced by the cumulative effect of data before the analysis period.

In previous studies [16,20-22,25], predicting graft failure has been pursued by taking an overlapping patient stratification approach, which means that each subsequent time cohort includes data from the previous cohort. This introduces a cumulative effect that is useful for predicting graft failure across a staggered time period. However, the overlapping patient stratification approach is ineffective in determining the influence of clinical features during a specific time period. Hence, the nonoverlapped cohort approach offers a novel mechanism to investigate the influence of clinical features within specific time windows. To the best of our knowledge, nonoverlapping cohorts have not been studied in the literature to develop prediction models or analyze the temporal influence of clinical features on kidney transplant outcomes.

This study is organized into five major sections: Methods presents the study's methodology; Results presents the results of the prediction; and Discussion discusses the significance of clinical features toward graft status prediction across different time cohorts and offers a conclusion and future research directions.

\section{Methods}

\section{Overview}

To predict graft survival over time and to analyze the influence of clinical features on graft survival, our data analytics methodology (Figure 1) comprised data preparation, feature engineering, prediction modeling, model evaluation, and analysis of changing relevance of features. 
Figure 1. Overview of our data analytics methodology. AUC: area under the curve; SMOTE: synthetic minority oversampling technique; UNOS: united network of organ sharing.

Step 1: Data preparation

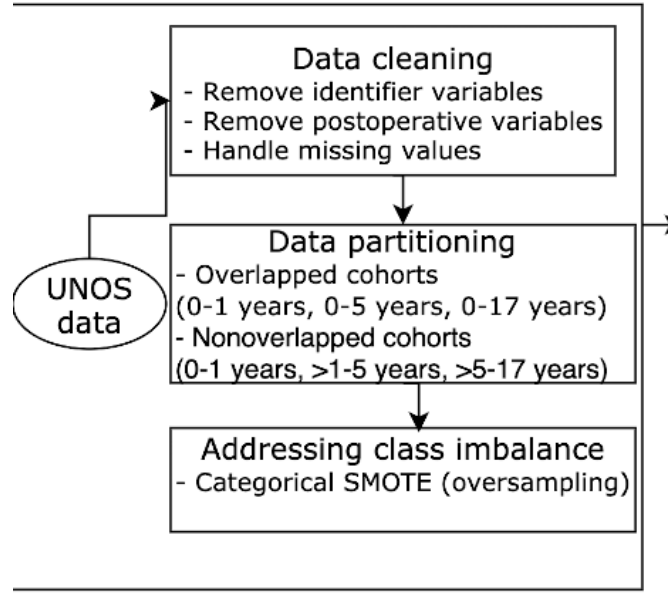

Step 2: Feature engineering

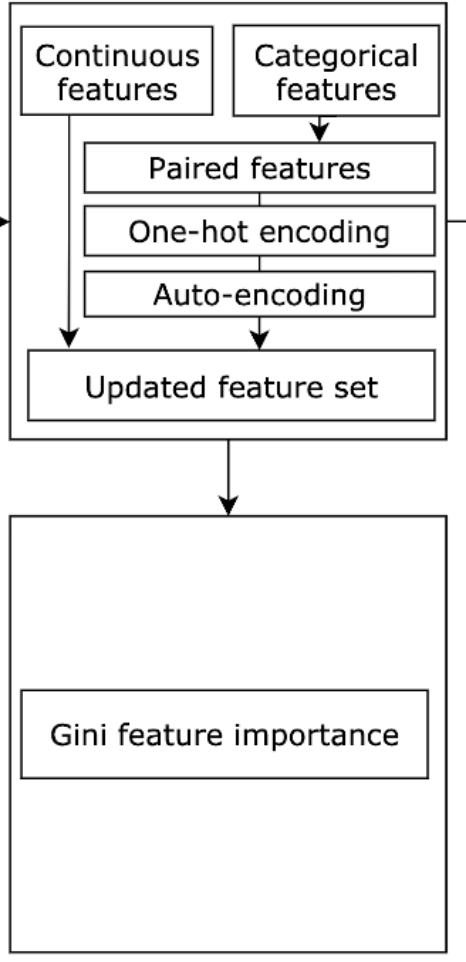

Step 5: Analysis of changing relevance of features
Step 3: Prediction modeling

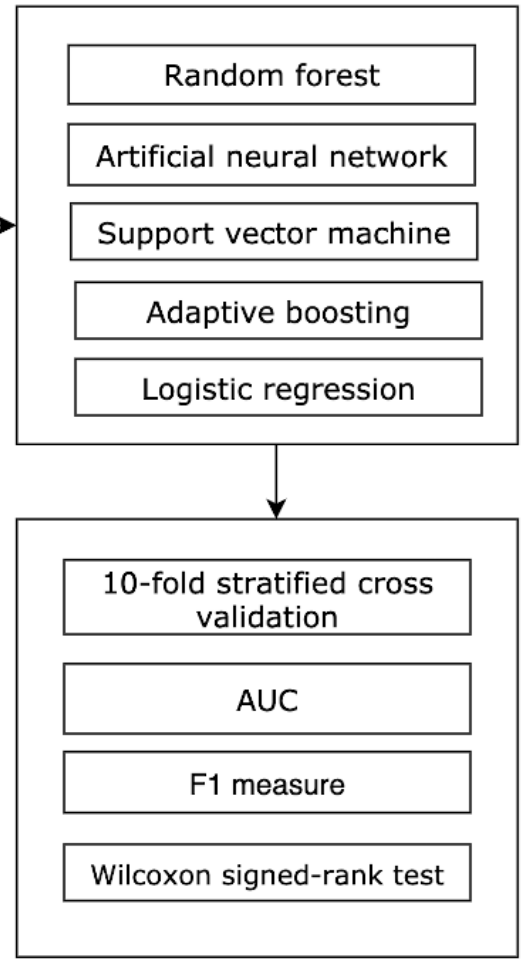

Step 4: Model evaluation

\section{Data Description}

This study used data from the Scientific Registry of Transplant Recipients (SRTR). The SRTR data system includes data on all donors, wait-listed candidates, and transplant recipients in the United States, submitted by the members of the Organ Procurement and Transplantation Network. The Health Resources and Services Administration and the US Department of Health and Human Services provided an overview of the activities of the Organ Procurement and Transplantation Network and SRTR contractors.
The data set provided pretransplant clinical features and outcomes of 277,316 kidney transplants between 2000 and 2017. Survival was reported in terms of graft outcome and patient status. For the purposes of this study, graft failure was defined as (1) graft loss or (2) death with a functioning graft.

We analyzed the data and used only complete cases (ie, no missing feature values), which comprised a total of 52,827 kidney transplants. Table 1 provides a list of the included clinical features and their descriptions used in our experiments. 
Table 1. List of clinical features used to train the prediction models.

\begin{tabular}{|c|c|c|}
\hline Feature description & Data type & Abbreviation \\
\hline Peak panel reactive antibody & Continuous & PKPRA \\
\hline Type of transplant & Categorical & REC_TX_PROCEDURE \\
\hline Any previous kidney transplant & Categorical & PREVKI \\
\hline Donor age & Continuous & DAGE \\
\hline Donor height & Continuous & DHT100 \\
\hline Recipient height & Continuous & RHT2100 \\
\hline Donor weight & Continuous & DWT \\
\hline Recipient weight & Continuous & RWT2 \\
\hline Donor creatinine level & Continuous & DONCREAT \\
\hline Expanded criteria donor & Categorical & ECD \\
\hline Donation after cardiac death & Categorical & DCD \\
\hline Donor hypertension & Categorical & DHTN2 \\
\hline Recipient hypertension & Categorical & RHTN \\
\hline Recipient BMI & Continuous & RBMI2 \\
\hline Donor BMI & Continuous & DBMI \\
\hline Cold ischemia time & Continuous & CIT \\
\hline Recipient age & Continuous & RAGETX \\
\hline Number of HLA antigen mismatches (paired) & Categorical & HLAMM \\
\hline Functional status of the recipient & Categorical & FUNCTSTAT \\
\hline Donor-recipient sex (paired) & Categorical & DRSEX \\
\hline Donor-recipient race (paired) & Categorical & DRRACE \\
\hline Donor-recipient age (paired) & Categorical & DRAGE \\
\hline Recipient cardiovascular disease & Categorical & RCVD \\
\hline Donor hepatitis $\mathrm{C}$ virus & Categorical & DHCV \\
\hline Recipient peripheral vascular disease & Categorical & RPVD \\
\hline Donor race & Categorical & DRACESIMP \\
\hline Recipient race & Categorical & RRACESIMP \\
\hline Recipient malignancy & Categorical & RMALIG \\
\hline Years on dialysis pretransplant & Continuous & VINTAGE \\
\hline Donor diabetes & Categorical & DDM \\
\hline Preemptive transplant & Categorical & PREEMPTIVE \\
\hline Recipient diabetes & Categorical & RDM2 \\
\hline Recipient coronary artery disease & Categorical & RCAD \\
\hline Simplified ESRD ${ }^{\mathrm{a}}$ diagnosis & Categorical & ESRDDXSIMP \\
\hline Donor-recipient $\mathrm{CMV}^{\mathrm{b}}$ (paired) & Categorical & DRCMV \\
\hline Donor-recipient height difference & Categorical & AHD1 \\
\hline Donor-recipient weight difference & Categorical & DRWT \\
\hline
\end{tabular}

${ }^{\mathrm{a}}$ ESRD: end-stage renal disease.

${ }^{\mathrm{b}} \mathrm{CMV}$ : cytomegalovirus. 


\section{Data Preparation}

Data preparation for learning the ML-based prediction models consisted of data cleaning, partitioning the data set into temporal cohorts, and addressing class imbalances.

\section{Data Cleaning}

Data cleaning involved removing (1) all patient identifying features (such as transplant ID, donor ID, and patient ID) [23], (2) post- and intraoperative features (such as delayed graft function and warm ischemia time) as we focused on pretransplant features to predict outcomes [23], (3) living donors $[15,26],(4)$ recipients below the age of 18 years $[14,27,28]$, and
(5) all sequential and en bloc transplants, both of which are atypical procedures that would not broadly apply to most deceased donor situations. These exclusion criteria were suggested by domain experts and also noted in prior studies $[9,28]$.

\section{Data Partitioning Into Temporal Cohorts}

Given the longitudinal data set, we generated two distinct data sets using traditional overlapping and our novel nonoverlapping patient stratification approaches to partition the data set into three temporal cohorts representing graft status at 1 year, $>1-5$ years, and 5-17 years (Figures 2 and 3).

Figure 2. Derivation of the overlapped cohorts.

\section{Overlapped cohorts}

Cohort 3

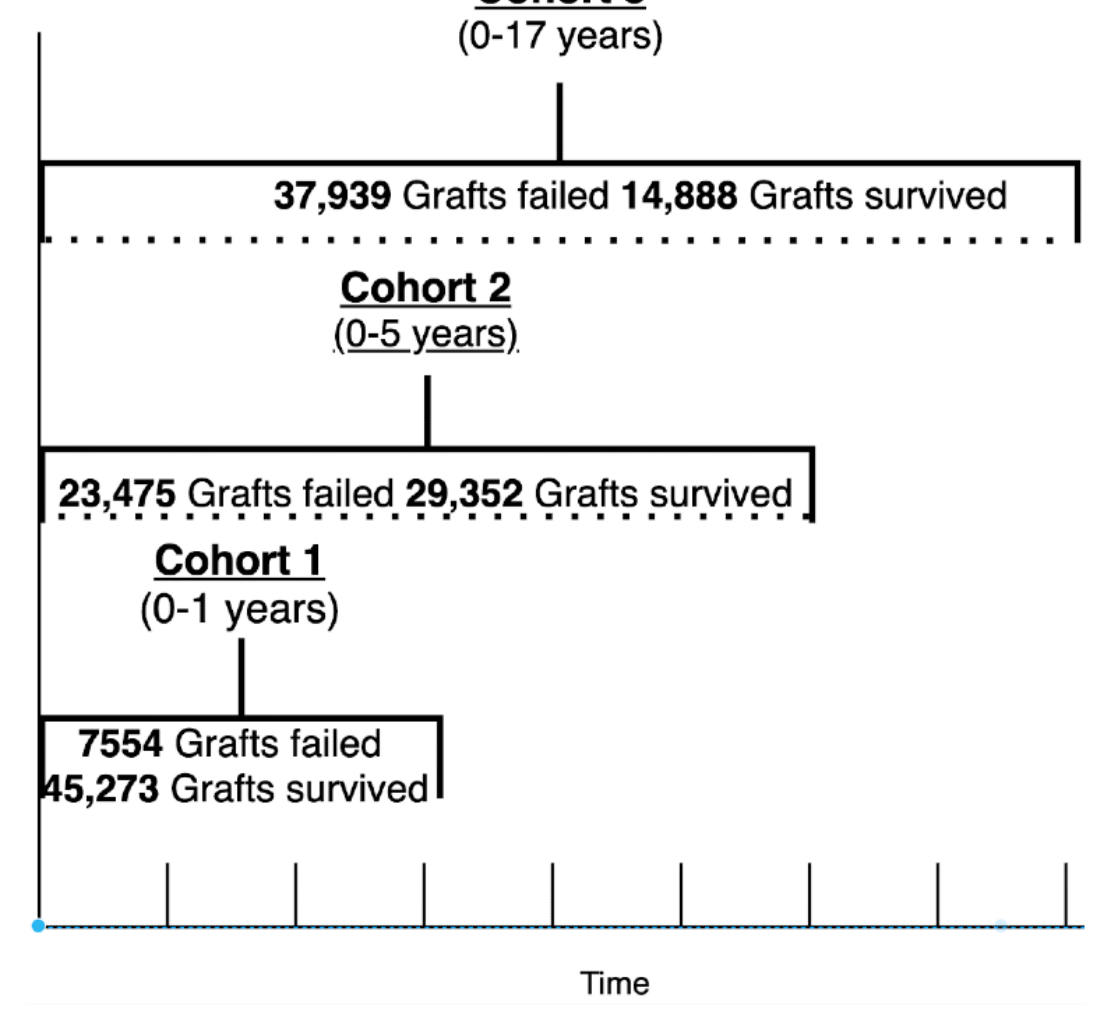

Figure 3. Derivation of the nonoverlapped cohorts.

\section{Nonoverlapped cohorts}

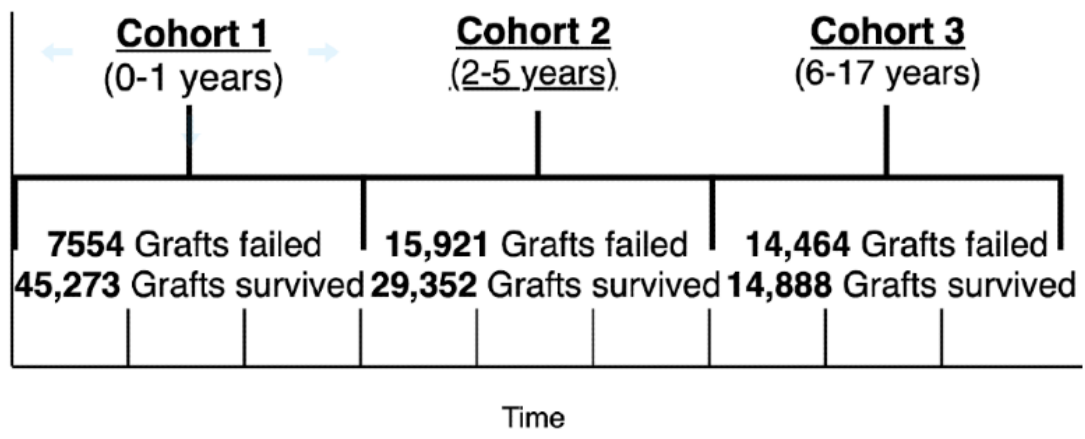

The overlapping patient stratification approach (used in previous graft status prediction studies) provides a cumulative analysis of graft outcomes up to a specific time point. In our study, the overlapping data stratification resulted in the following three 
cohorts: cohort 1 , spanning from year to year $_{1}$, which reported graft outcomes (ie, graft failure or survival) during this period; cohort 2, which reported graft status from year to year $_{5}$ and overlapped with cohort 1 such that it included the patients in cohort 1; and cohort 3 , which reported graft status from year ${ }_{0}$ to year ${ }_{10+}$, thus overlapping with both earlier cohorts. As per the overlapping approach, a graft failure in the preceding cohort was also counted in the proceeding cohort.

Our nonoverlapping patient stratification approach yielded three cohorts: cohort 1 , spanning year $_{0}$ to year ${ }_{1}$, reporting graft outcomes in this period; cohort 2 , spanning year to year $_{5}$ and reporting graft outcomes only in this period, resulting in the exclusion of graft failures reported in cohort 1 and only reporting the graft outcomes of patients who survived cohort 1; and cohort 3 , spanning year to year $_{10+}$, reporting the graft outcomes of patients who survived cohort 2. In a nonoverlapping cohort approach, there was no looking back beyond the cohort's starting point, as such a graft failure in the preceding cohort was not counted in the proceeding cohort.

When partitioning the data into cohorts, we accounted for the presence of censored data, that is, the lack of information about the occurrence of an adverse event for a surviving patient. There is no concrete method to determine survivors when confronted with censored data. We initially assumed that those patients who did not fail in a certain cohort could be presumed as survivors. However, this assumption led to two problems: (1) it included censored patients who might have experienced graft failure during the study, and (2) it led to a severe class imbalance between the graft failure and surviving patients. To overcome these problems, we took a two-phase heuristic approach to remove the censored observations to identify survivors in each cohort. First, we removed all the censored observations from the cohort being analyzed and labeled all the remaining instances as survived. For instance, the censored data that were removed for cohort 2 were all instances with a missing outcome by the end of cohort 2. The remaining instances were considered to have survived. The first phase of our approach reduced the number of censored observations, but the surviving observations were still relatively high compared with the graft failure cases in each cohort. In the second phase, we applied the approach of Topuz et al [15] to further refine our surviving class by removing all the instances that were deemed as surviving for less than 8 years from the date of transplant. This two-phase approach to account for censored data is summarized in Equation 1 below, where we estimated the number of survivors in each cohort.

$$
S_{i}=\left\{\begin{array}{c}
\text { Yes if }(\text { days } \geq i * 365 \text { \& graft } \text { status }==\text { failed }) \\
+(\text { days } \geq 2920 \text { \& graft status } !=\text { failed }) \\
\text { No otherwise }
\end{array}\right.
$$

The first part of this equation illustrates the first phase of the proposed approach. The $i$ in the equation is the ending year of our defined cohorts, that is, 1,5 , and 17. The equation first calculates the total number of graft failures that occurred after the end of the cohort. This fraction of instances was considered as confirmed survivors for the cohort under analysis. The second part of the equation deals with the second phase of the approach. It attempts to identify the potential survivors from the censored data by removing all the observations that did not have a graft failure within 8 years (2920 days) following a transplant. Table 2 shows the patient distribution across the three time cohorts.

Table 2. Number of failed and survived transplants in overlapped and nonoverlapped cohorts.

\begin{tabular}{lllllll}
\hline Cohort & Overlapping & & & \multicolumn{2}{l}{ Nonoverlapping } \\
& Count, $\mathrm{n}$ & Failed, n (\%) & Survived, n (\%) & Count, $\mathrm{n}$ & Failed, n (\%) & Survived, n (\%) \\
\hline 1 & 52,827 & $7554(14.3)$ & $45,273(85.7)$ & 52,827 & $7554(14.3)$ & $45,273(85.7)$ \\
2 & 52,827 & $23,475(44.44)$ & $29,352(55.56)$ & 45,273 & $15,921(35.17)$ & $29,352(64.83)$ \\
3 & 52,827 & $37,939(71.82)$ & $14,888(28.18)$ & 29,352 & $14,464(49.28)$ & $14,888(50.72)$ \\
\hline
\end{tabular}

\section{Addressing Class Imbalance}

Our data set had two outcomes: the presence or absence of graft failure. There was a significant class imbalance whereby the graft failure had a significantly lower number of instances compared with graft survival. Techniques such as Synthetic Minority Oversampling Technique (SMOTE) and random undersampling have been widely used in the literature [18,29] for class imbalance. We applied SMOTE for Nominal and Continuous features [30], which is a variant of SMOTE specifically developed to handle a mix of categorical and numerical data, on all cohorts to achieve a reasonable class balance. To balance the minority class (ie, mostly graft failure), we would need to generate $600 \%$ additional synthetic samples (at least for cohort 1), which would have led to overfitting. Therefore, we doubled our minority class to achieve a workable class balance to prevent the overfitting of the classification models. As cohort 2 of overlapped stratification and cohort 3 of nonoverlapped stratification had a class balance, they were not considered for oversampling.

\section{Feature Engineering}

This step involved both the removal and construction of features with the intent to reduce the dimensionality of the feature space.

\section{Paired Features}

A set of paired features was constructed by combining the related features. Typically, graft predictions use discrete individual donor and recipient features. We examined the underlying correlation between the donor and recipient features and paired the highly related features to generate new paired features. The following four donor and recipient features were generated: sex, age, CMV (cytomegalovirus), and race. In addition, three types of HLA Antigen Mismatch features-ie, HLA Antigen Mismatch at the A Locus, HLA Antigen Mismatch at the B Locus, and HLA Antigen Mismatch at the 
DR Locus were also combined into a single HLA Antigen Mismatch feature.

\section{One-Hot Encoding}

We transformed the categorical features into multiple dummy features to make them compatible with the stacked autoencoders, which cannot process categorical features. In addition, it was also a necessary operation because of the functional constraint of the scikit-learn library [31].

\section{Stacked Autoencoders}

Finally, we used 86 transformed categorical features as inputs to the stacked autoencoders for feature reduction to subsequently train the ML prediction models. Continuous features were also initially considered as a part of the input vector to stacked autoencoders (Table S1 in Multimedia Appendix 1), but preliminary model training returned better results with pristine continuous features; hence, no modification was performed for them while training the prediction models. It should be noted that the resultant features from the stacked autoencoders were only used for training the prediction models and not for the analysis of the changing relevance of features over time.

After testing with different configuration settings provided in the Keras framework [32], the stacked autoencoders were set up as a 13-layer architecture consisting of 12 dense layers and one dropout layer set at the very beginning of the network with a dropout rate of 0.05 . The sigmoid activation function was used throughout the dense layers, with Adam as the optimizer and binary cross entropy as the loss function with 500 epochs and 700-900 batch sizes. The middle layer of the autoencoder was finally trained with 12 neurons and 30 neurons for cohort 1 and the remaining cohorts, respectively. The feature space was reduced by more than 50 dummy features.

\section{Learning Classification-Based Graft Survival Prediction Models}

\section{Overview}

Prediction was pursued as a binary classification problem, where the prediction output represents the graft outcome for a given patient in terms of the class label, graft failure or survived. We investigated four different ML-based classification models for each time cohort (ie, cohorts 1-3). Given that logistic regression (LR) has been widely used in prior studies to develop graft prediction models [29,33], we trained an LR model as a baseline to compare the predictive performance of our ML prediction models.

All classification models were trained using a 10-fold stratified cross-validation training approach. The stratification ensured that outcome class ratio in each fold is maintained to avoid any sampling bias that may affect the classification results. We mainly used the scikit-learn library [31] to train the below-mentioned classification models with the parameter settings listed in Table 3. Hyperparameters were optimized using a random search. The different parameters that were tested during the random search are provided in Table $\mathrm{S} 2$ in Multimedia Appendix 1. 
Table 3. Algorithmic settings for the classifiers.

\begin{tabular}{|c|c|}
\hline Method, Hyperparameter & Values \\
\hline \multicolumn{2}{|l|}{$\mathbf{R F}^{\mathbf{a}}$} \\
\hline Number of estimators & 200 \\
\hline Class weight & Balanced \\
\hline Criterion & Gini \\
\hline Maximum depth & 9 \\
\hline Minimum samples split & 2 for cohort $1 ; 3$ for the rest \\
\hline Maximum features & 14 \\
\hline \multicolumn{2}{|l|}{ Support vector machine } \\
\hline $\mathrm{C}^{\mathrm{b}}$ & 50 \\
\hline Gamma & Auto, scale \\
\hline Decision function shape & One versus rest \\
\hline Kernel & Radial \\
\hline \multicolumn{2}{|l|}{ Artificial neural network } \\
\hline Solver & Adam \\
\hline Learning rate & Adaptive \\
\hline Activation & Logistic \\
\hline Alpha & $1 \mathrm{e}-2,1 \mathrm{e}-6$ \\
\hline Hidden layers & $4: 70,35,30,15 ; 5: 60,30,30,15,10$ \\
\hline \multicolumn{2}{|l|}{ Adaptive boosting } \\
\hline Base learner & $\mathrm{RF}$ \\
\hline Number of estimators & 401 \\
\hline Learning rate & 1 \\
\hline Algorithm & Samme.R \\
\hline \multicolumn{2}{|l|}{ Logistic regression } \\
\hline Penalty & 12 \\
\hline $\mathrm{C}$ & 10 \\
\hline Class weight & Balanced \\
\hline Max iteration & 1000 \\
\hline Solver & Sag \\
\hline
\end{tabular}

${ }^{\mathrm{a}} \mathrm{RF}$ : random forest.

${ }^{\mathrm{b}} \mathrm{C}$ : regularization parameter.

\section{Random Forest}

Random forest (RF) was used as both a standalone classifier and a base learner for the adaptive boosting (AdaBoost) algorithm. It has been widely used to predict survival data [26,27].

\section{AdaBoost}

The AdaBoost algorithm was applied to two weak learners, RF and LR. The study by Thongkam et al [34] used this algorithm on a breast cancer data set, where it outperformed all single classifiers. In our experiments, LR did not perform well; therefore, we did not pursue it. RF, with the optimized hyperparameters, was used to train the boosting classifier with a number of estimators and learning rates.

\section{Artificial Neural Network}

A backpropagation algorithm was used to train a neural network-based binary classifier. Generally, artificial neural networks (ANNs) perform well on survival data sets [30,35].

\section{Support Vector Machines}

Classification models using support vector machines (SVMs) have been applied to predict survival data $[29,31,32]$. To train the SVM, we experimented with different kernels, that is, linear, radial, sigmoid, and polynomial kernels. The linear and sigmoid kernels provided the lowest prediction scores; therefore, we did 
not use them further. A polynomial kernel with degree 2 yielded suboptimal results, and the SVM model could not converge for degree 3. The radial basis kernel was the most effective for learning the classification model.

\section{Calculating Feature Importance Over Time}

The nonoverlapped time cohorts were used to calculate the feature importance scores to understand the changing relevance of features over time. We calculated these scores by training an $\mathrm{RF}$ classifier on the complete data set. The scores were calculated using Gini. Feature influence scores were used to understand the effect of features over the three cohorts.

\section{Results}

\section{Overview}

Below, we present the prediction performance of the four ML classifiers using both overlapped and nonoverlapped cohorts. As LR has been extensively used to predict time-to-event in organ transplant studies [16,29], it is used as a comparator classifier to the ML-based classifiers. The prediction performance of each of the best-trained classifiers (SVM, RF,
AdaBoost, ANN, and LR), covering the three different time-to-event periods for both the original and reduced feature sets, were evaluated using 10-fold stratified cross validation for both overlapped and nonoverlapped cohorts. The results of each classifier were examined using the area under the curve (AUC) and F1 scores. The AUC score was used as the main performance evaluation metric to select the best model in each cohort and to make comparisons with similar studies. For our purpose, the ideal prediction model provides the best accuracy for graft failure. Therefore, to further substantiate the selection of the best model, we also evaluated the F1 score for graft failures. In cases where the AUC score was the same for different models, preference was given to the model with the highest F1 score.

\section{Analysis of Feature Engineering}

Table 4 presents the results of feature engineering, whereby the prediction scores of all classifiers were obtained using both the original feature set and the reduced set. The reduced set consists of original continuous features and latent features returned by the stacked autoencoders. Cohort 1 for overlapped and nonoverlapped cohorts was the same; hence, the results were presented only once to avoid unnecessary duplication. 
Table 4. Area under the curve comparison-all features with auto-encoded features.

\begin{tabular}{|c|c|c|c|c|}
\hline \multirow[t]{2}{*}{ Cohort } & \multicolumn{2}{|l|}{ Overlapped } & \multicolumn{2}{|l|}{ Nonoverlapped } \\
\hline & All features (\%) & $\begin{array}{l}\text { Continuous+auto-en- } \\
\text { coded features }(\%)\end{array}$ & All features (\%) & $\begin{array}{l}\text { Continuous+auto-en- } \\
\text { coded features }(\%)\end{array}$ \\
\hline \multicolumn{5}{|l|}{ Cohort 1} \\
\hline $\mathrm{SVM}^{\mathrm{a}}$ & 80 & 82 & $N / A^{b}$ & N/A \\
\hline AdaBoost ${ }^{\mathrm{c}}$ & 76 & 78 & N/A & N/A \\
\hline $\mathrm{RF}^{\mathrm{d}}$ & 68 & 70 & N/A & N/A \\
\hline $\mathrm{ANN}^{\mathrm{e}}$ & 62 & 61 & N/A & N/A \\
\hline$L R^{f}$ & 62 & 62 & N/A & N/A \\
\hline \multicolumn{5}{|l|}{ Cohort 2} \\
\hline SVM & 63 & 66 & 53 & 53 \\
\hline AdaBoost & 67 & 69 & 64 & 60 \\
\hline $\mathrm{RF}$ & 62 & 65 & 65 & 67 \\
\hline ANN & 62 & 62 & 62 & 62 \\
\hline LR & 62 & 62 & 64 & 61 \\
\hline \multicolumn{5}{|l|}{ Cohort 3} \\
\hline SVM & 73 & 80 & 68 & 65 \\
\hline AdaBoost & 76 & 81 & 68 & 64 \\
\hline $\mathrm{RF}$ & 72 & 75 & 68 & 66 \\
\hline ANN & 73 & 72 & 68 & 65 \\
\hline LR & 69 & 69 & 62 & 64 \\
\hline
\end{tabular}

${ }^{\mathrm{a}} \mathrm{SVM}$ : support vector machine.

${ }^{\mathrm{b}}$ N/A: not applicable.

${ }^{\mathrm{c}}$ AdaBoost: adaptive boosting.

${ }^{\mathrm{d}} \mathrm{RF}$ : random forest.

${ }^{\mathrm{e}} \mathrm{ANN}$ : artificial neural network.

${ }^{\mathrm{f}}$ LR: logistic regression.

The AUC scores (Table 4) show that prediction models for overlapped cohorts trained with auto-encoded features improved the prediction performance as compared with the prediction models trained using the original feature set. However, it was the opposite for nonoverlapped cohorts. Interestingly, the traditional approach of overlapped cohorts performed better with both the original and reduced feature sets compared with the nonoverlapped cohorts. Except for RF in cohort 2 of nonoverlapped cohorts, which showed slightly better performance $(67 \%)$ when compared with its overlapped counterpart (65\%), all other prediction models had better AUC scores with the traditional overlapping cohort approach.

Therefore, for further analysis, we proceeded with overlapping cohorts only.

Although ANN and LR (the baseline model) showed no significant improvement across all three cohorts, the results confirmed the effectiveness of our deep learning architecture of stacked autoencoders for feature selection. For the subsequent prediction modeling analysis, we used the reduced feature set.

\section{Analysis of Prediction Performance of ML Models}

Table 5 presents the prediction performance of the classifiers for each cohort in terms of AUC, F1 scores, recall, and precision, with SD for the 10-fold classification. 
Table 5. Prediction performance of the machine learning classifiers across three different temporal cohorts using the overlapped patient stratification ${ }^{\mathrm{a}}$.

\begin{tabular}{|c|c|c|c|c|}
\hline \multirow[t]{2}{*}{ Cohort } & \multicolumn{4}{|l|}{ Auto-encoded feature set } \\
\hline & $\operatorname{AUC}^{\mathrm{b}}(\%)$, mean $(\mathrm{SD})$ & $\mathrm{F} 1(\%)$, mean $(\mathrm{SD})$ & Recall $(\%)$, mean (SD) & Precision $(\%)$, mean (SD) \\
\hline \multicolumn{5}{|l|}{ Cohort 1} \\
\hline$S V M^{\mathrm{c}}$ & $82(0.01)$ & $61(0.01)$ & $49(0.01)$ & $90(0.01)$ \\
\hline AdaBoost $^{\mathrm{d}}$ & $78(0.01)$ & $56(0.01)$ & $95(0.01)$ & $35(0.01)$ \\
\hline $\mathrm{RF}^{\mathrm{e}}$ & $70(0.009)$ & $45(0.001)$ & $47(0.01)$ & $41(0.01)$ \\
\hline $\mathrm{ANN}^{\mathrm{f}}$ & $61(0.01)$ & $5(0.001)$ & $42(0.01)$ & $6(0.004)$ \\
\hline $\mathrm{LR}^{\mathrm{g}}$ & $62(0.008)$ & $39(0.009)$ & $58(0.01)$ & $29(0.04)$ \\
\hline \multicolumn{5}{|l|}{ Cohort 2} \\
\hline SVM & $66(0.006)$ & $53(0.01)$ & $55(0.01)$ & $60(0.01)$ \\
\hline AdaBoost & $69(0.01)$ & $63(0.01)$ & $64(0.003)$ & $63(0.004)$ \\
\hline $\mathrm{RF}$ & 65 (0.009) & $62(0.01)$ & $62(0.01)$ & $61(0.01)$ \\
\hline ANN & $63(0.007)$ & $60(0.04)$ & $55(0.09)$ & $60(0.01)$ \\
\hline LR & $62(0.008)$ & $59(0.009)$ & $58(0.01)$ & $60(0.004)$ \\
\hline \multicolumn{5}{|l|}{ Cohort 3} \\
\hline SVM & $80(0.005)$ & $83(0.003)$ & $76(0.003)$ & $96(0.003)$ \\
\hline AdaBoost & $81(0.01)$ & $81(0.004)$ & $76(0.003)$ & $86(0.01)$ \\
\hline $\mathrm{RF}$ & $75(0.008)$ & $75(0.006)$ & $75(0.01)$ & $73(0.01)$ \\
\hline ANN & $72(0.007)$ & $68(0.005)$ & $81(0.03)$ & $69(0.01)$ \\
\hline LR & $69(0.001)$ & 77 (0.009) & $70(0.01)$ & $70(0.001)$ \\
\hline
\end{tabular}

${ }^{\mathrm{a}}$ Italics show the classifiers with the highest performance among the three cohorts.

${ }^{\mathrm{b}} \mathrm{AUC}$ : area under the curve.

${ }^{\mathrm{c}} \mathrm{SVM}$ : support vector machine.

${ }^{\mathrm{d}}$ AdaBoost: adaptive boosting.

${ }^{\mathrm{e}} \mathrm{RF}$ : random forest.

${ }^{f}$ ANN: artificial neural network.

${ }^{\mathrm{g}}$ LR: logistic regression.

The classifiers performed differently across the three SD across the different folds was nominal, confirming the cohorts-SVM offered the highest prediction performance for stability of the classifiers. Figure 4 shows the receiver operating short-term predictions, that is, for cohort 1, whereas AdaBoost characteristic curves for the best models from each cohort. offered the highest performance for the remaining cohorts. The

Figure 4. Receiver operating characteristic curves for support vector machine, adaptive boosting, and adaptive boosting for the three cohorts, respectively (left to right). AUC: area under the curve; ROC: receiver operating characteristic.
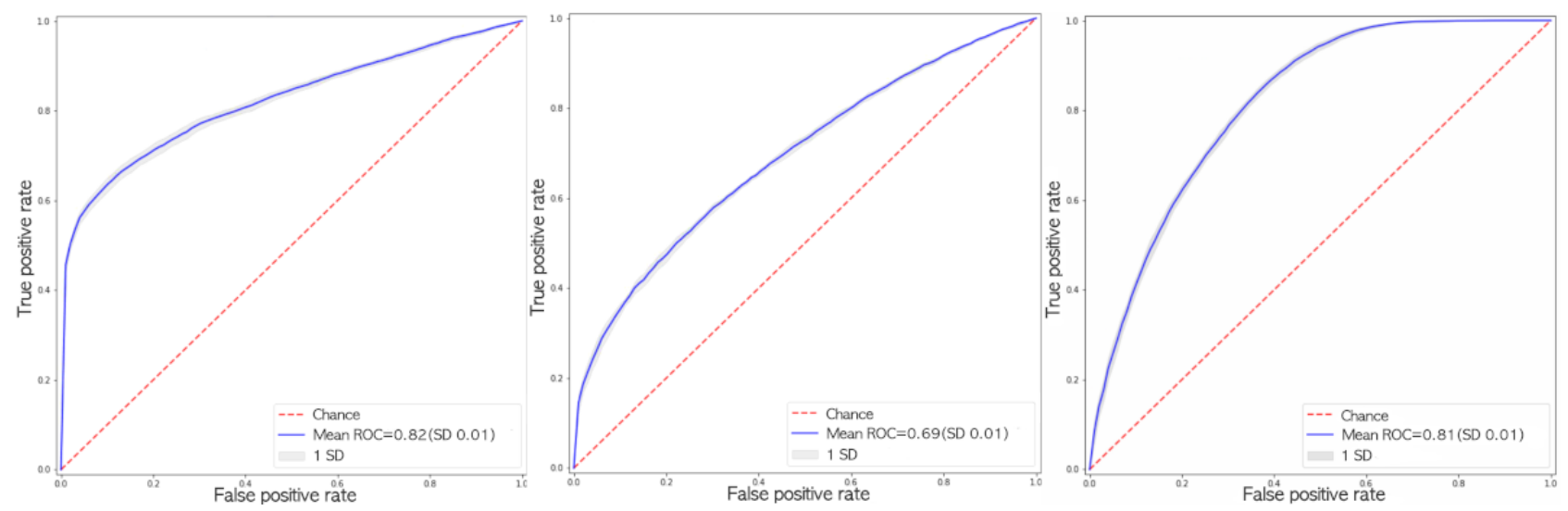
To further investigate the prediction efficacy of the ML-based classifiers, we evaluated the prediction performance of the best-performing classifier for all three cohorts by testing the prediction of graft failure events by a classifier trained for a specific cohort with data from other cohorts, that is, testing the classifier for cohort 2 with randomly selected data from cohorts 1 and 3 . The underlying assumption is that the classifier should not produce good prediction results for data from other cohorts. As this evaluation considers survivors across progressive cohorts, we used the F1 score to measure prediction performance. A sound prediction model for cohort 2 will give a high graft failure prediction score for data from cohort 1 but a low prediction score for data from cohort 3 , the rationale being that the overlapping cohort 2 classifier is trained on graft failure cases in both cohorts 1 and 2. Therefore, the prediction model for cohort 2 should give a high prediction score for predicting graft failures from year 0 to year ${ }_{5}$, but when applied to cohort 3 , the cohort 2 prediction model would be unable to predict graft failure as it has not been trained on cohort 3 data. Table 6 provides the cross-cohort prediction scores for the best classifiers for each cohort.

Table 6. Prediction performance (F1 scores) for cross-cohort predictions using overlapped cohorts.

\begin{tabular}{llll}
\hline Model & Cohort 1 & Cohort 2 & 0.42 \\
SVM $^{\mathrm{a}}$ (cohort 1) & 0.6 & 0.87 & 0.29 \\
AdaBoost $^{\mathrm{b}}$ (cohort 2) & 0.79 & 0.58 \\
AdaBoost (cohort 3) & 0.72 & 0.75 & 0.87 \\
\hline
\end{tabular}

${ }^{\mathrm{a}} \mathrm{SVM}$ : support vector machine.

${ }^{\mathrm{b}}$ AdaBoost: adaptive boosting.

\section{Results of Wilcoxon Signed-Rank Test}

To determine if the prediction differences between the different models were statistically significant, we used the Wilcoxon signed-rank test to compare the scores between different models. Because the best scores in each cohort were usually produced by SVM and AdaBoost models, the Wilcoxon signed-rank test was conducted with each combination of these models with the other models.

Table 7 shows the results based on the F1 score, and the $P$ values between the models were quite small and less than the threshold value of $P=.05$, confirming that the performance difference is statistically significant.

Table 7. The results for Wilcoxon signed-rank test (F1).

\begin{tabular}{|c|c|c|c|c|c|}
\hline \multirow[t]{2}{*}{ Cohort } & \multicolumn{5}{|l|}{$P$ value $(\mathrm{F} 1)$} \\
\hline & SVM $^{\mathrm{a}}$-AdaBoost ${ }^{\mathrm{b}, \mathrm{c}}$ & SVM-ANN ${ }^{\mathrm{d}, \mathrm{e}}$ & $S V M-R F^{f, g}$ & AdaBoost-RF ${ }^{\mathrm{h}}$ & AdaBoost-ANN ${ }^{\mathrm{i}}$ \\
\hline Cohort 1 & .003 & .003 & .003 & .003 & .003 \\
\hline Cohort 2 & .003 & .003 & .003 & .003 & .03 \\
\hline Cohort 3 & $<.001$ & $<.001$ & $<.001$ & $<.001$ & $<.001$ \\
\hline
\end{tabular}

${ }^{\mathrm{a}} \mathrm{SVM}$ : support vector machine.

${ }^{\mathrm{b}}$ AdaBoost: adaptive boosting.

${ }^{\mathrm{c}} \mathrm{H}_{\mathrm{O}}$ (null hypothesis): $\mathrm{SVM}=\mathrm{AdaBoost} ; \mathrm{H}_{\mathrm{a}}$ (alternative hypothesis): $\mathrm{SVM} \neq \mathrm{AdaBoost}$.

$\mathrm{d}_{\mathrm{ANN}}$ : artificial neural network

${ }^{\mathrm{e}} \mathrm{H}_{\mathrm{O}}: \mathrm{SVM}=\mathrm{EANN} ; \mathrm{H}_{\mathrm{a}}: \mathrm{SVM} \neq \mathrm{EANN}$.

${ }^{\mathrm{f}} \mathrm{RF}$ : random forest.

$\mathrm{g}_{\mathrm{H}_{\mathrm{O}}}: \mathrm{SVM}=\mathrm{RF} ; \mathrm{H}_{\mathrm{a}}: \mathrm{SVM} \neq \mathrm{RF}$.

${ }^{\mathrm{h}} \mathrm{H}_{\mathrm{o}}:$ AdaBoost=RF; $\mathrm{H}_{\mathrm{a}}:$ AdaBoost $\neq \mathrm{RF}$.

${ }^{\mathrm{i}} \mathrm{H}_{\mathrm{o}}:$ AdaBoost $=\mathrm{ANN} ; \mathrm{H}_{\mathrm{a}}:$ AdaBoost $\neq \mathrm{ANN}$.

\section{Analysis of the Influence of Clinical Features Over Time Toward Graft Status Prediction}

\section{Overview}

The second objective of this research is to analyze the influence of clinical features on the prediction of graft survival over different periods. The intent was to understand the factors responsible for graft survival at different periods after transplant. The nonoverlapped cohorts $(0-1$ years, $>1-5$ years, and $>5-17$ years following a transplant) were used to ensure that there was no cascading influence of the features over time. For comparison purposes, we also examined the feature importance for overlapping cohorts. The feature importance scores represent the relative importance of the feature among all features, that is, the total of all the features' importance scores add up to $100 \%$; hence, if one feature gains a higher importance score, it will be at the expense of the importance score of other features. 
Figures 5 and 6 illustrate the individual feature importance respectively. scores across all the nonoverlapped and overlapped cohorts,

Figure 5. Changing relevance of features based on nonoverlapped time cohorts.

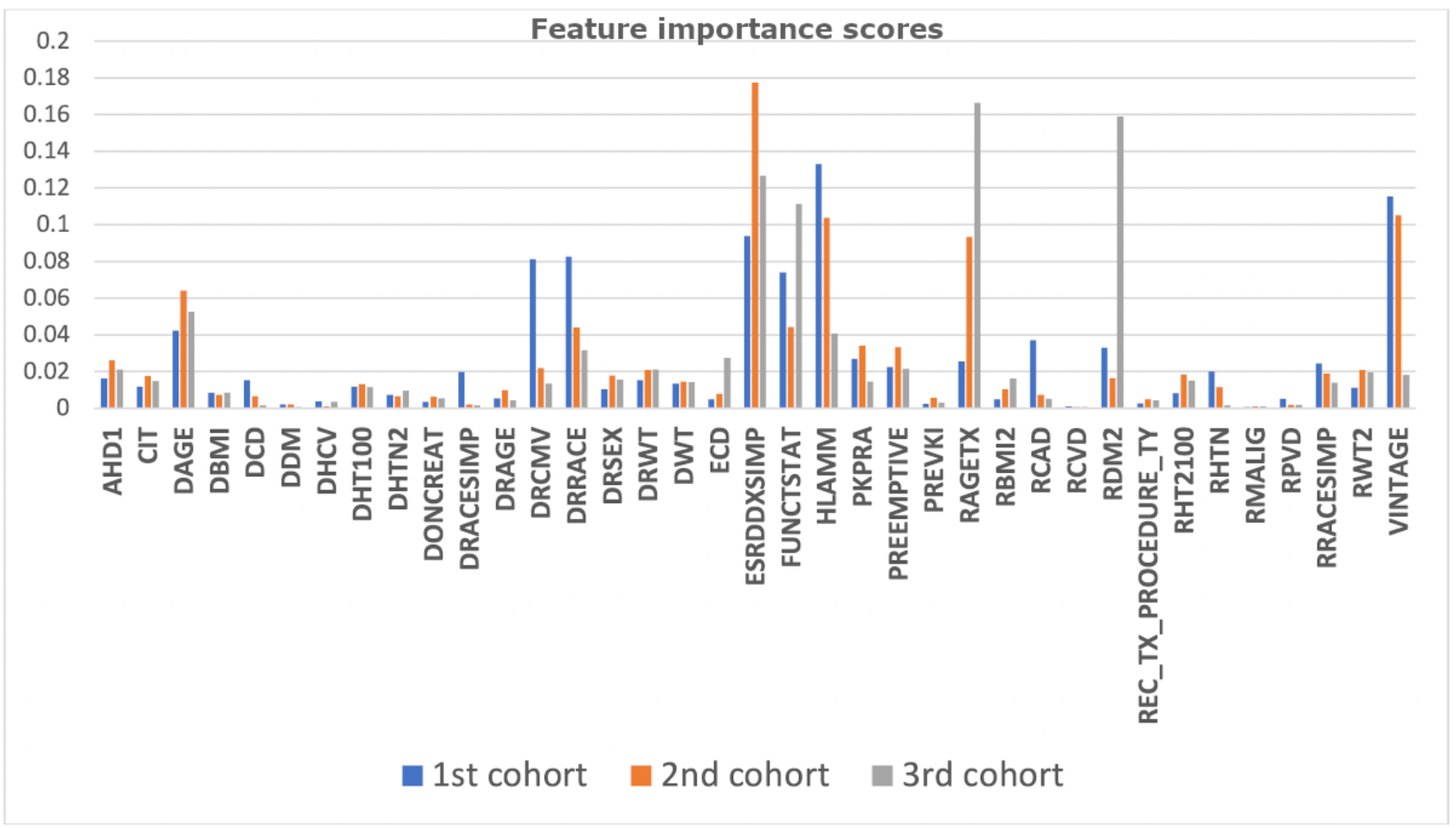

Figure 6. Changing relevance of features based on overlapped time cohorts.

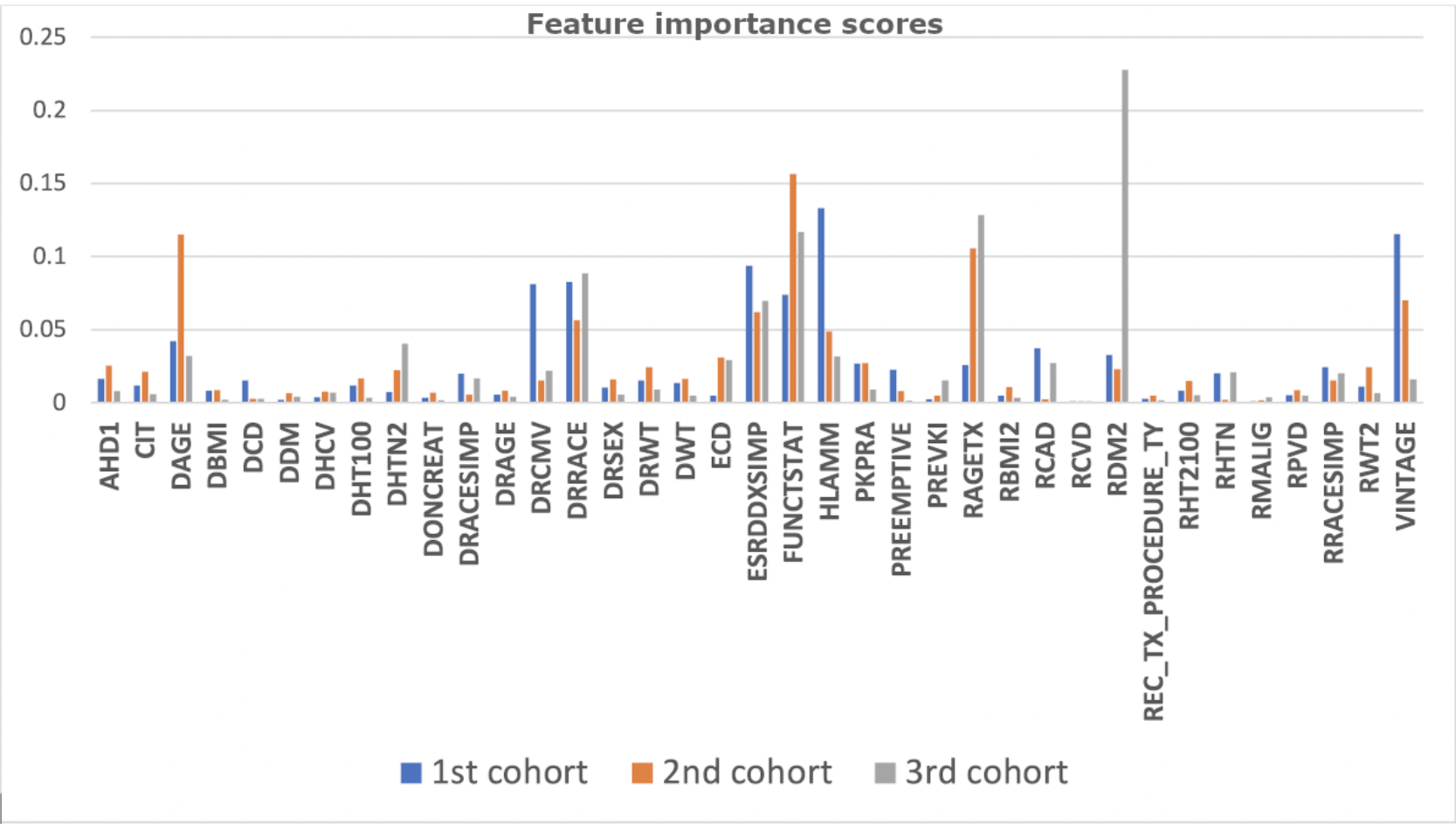

In general, the top $10 \%$ of the important features remained consistent in both the nonoverlapped and overlapped cohorts; however, we note that the nonoverlapped cohorts identified a larger group of important features. For instance, peak panel reactive antibody (Pkpra) and pre-emptive recipient status
(Preemptive) had negligible importance in overlapping cohorts but were important during the 2-5 years and 6-17 years in the nonoverlapping cohorts. Table 8 shows the feature importance across the three cohorts. 
Table 8. Ranking of the top- 10 features across the time cohorts with feature importance scores ${ }^{\mathrm{a}}$.

\begin{tabular}{|c|c|c|c|c|c|}
\hline \multirow[t]{2}{*}{ Rank } & \multirow{2}{*}{$\begin{array}{l}\text { Cohort } 1 \text {, feature, relative } \\
\text { score }(\%)\end{array}$} & \multicolumn{2}{|l|}{ Cohort 2} & \multicolumn{2}{|l|}{ Cohort 3} \\
\hline & & Feature, relative score $(\%)$ & $\begin{array}{l}\text { Importance }(\%) \text {, rank } \\
\text { change }\end{array}$ & $\begin{array}{l}\text { Feature, relative score } \\
(\%)\end{array}$ & $\begin{array}{l}\text { Importance }(\%) \text {, rank } \\
\text { change }\end{array}$ \\
\hline 1 & $\operatorname{HLAMM}^{\mathrm{b}}(13)$ & ESRDDXSIMP $^{\mathrm{c}}(18)$ & $+100,+2$ & RAGETX $^{\mathrm{d}}(16)$ & $+77,+3$ \\
\hline 2 & $\operatorname{VINTAGE}^{\mathrm{e}}(12)$ & VINTAGE (11) & $-8,0$ & $\operatorname{RDM}^{\mathrm{f}}(16)$ & $>+100,>+10$ \\
\hline 3 & ESRDDXSIMP (9) & HLAMM (10) & $-23,-2$ & ESRDDXSIMP (13) & $-27,-2$ \\
\hline 4 & $\operatorname{DRCMV}^{\mathrm{g}}(8)$ & RAGETX (9) & $+78,+6$ & FUNCTSTAT $^{\mathrm{h}}$ (12) & $>+100,+3$ \\
\hline 5 & $\operatorname{DRRACE}^{\mathrm{i}}(8)$ & $\operatorname{DAGE}^{\mathrm{j}}(6)$ & $50,+2$ & DAGE (5) & $-16,0$ \\
\hline 6 & FUNCTSTAT (7) & DRRACE (4) & $-100,-1$ & DRRACE (3) & $-25,0$ \\
\hline 7 & DAGE (4) & FUNCTSTAT (4) & $-75,-1$ & $\operatorname{ECD}^{\mathrm{k}}(3)$ & $>+100,>+3$ \\
\hline 8 & $\operatorname{RCAD}^{1}(4)$ & $\operatorname{PKPRA}^{\mathrm{m}}(3)$ & $+50,>+3$ & VINTAGE (2) & $>-100,-6$ \\
\hline 9 & RDM2 (3) & PREEMPTIVE $^{\mathrm{n}}(3)$ & $+50,>+1$ & PREEMPTIVE (2) & $-33,0$ \\
\hline 10 & RAGETX (2) & DRCMV (2) & $-75,-6$ & RWT2 (2) & 0,0 \\
\hline Rest & Rest (31) & Rest (30) & Rest (30) & Rest (26) & Rest (26) \\
\hline
\end{tabular}

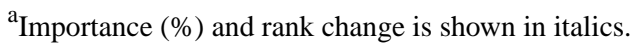

${ }^{b}$ HLAMM: HLA antigen mismatch.

${ }^{\mathrm{c}}$ ESRDDXSIMP: simplified end-stage renal disease diagnosis.

${ }^{\mathrm{d}}$ RAGETX: recipient age.

${ }^{\mathrm{e}}$ VINTAGE: number of years on dialysis before transplant.

${ }^{\mathrm{f}} \mathrm{RDM} 2$ : recipient diabetes status.

gDRCMV: donor-recipient cytomegalovirus.

${ }^{h}$ FUNCTSTAT: functional status of the recipient.

${ }^{\mathrm{i} D R R A C E}$ : donor-recipient race.

${ }^{\mathrm{j}}$ DAGE: donor age.

${ }^{\mathrm{k}} \mathrm{ECD}$ : expanded criteria donor.

${ }^{1} \mathrm{RCAD}$ : recipient coronary artery disease.

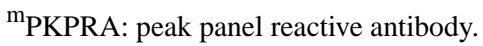

${ }^{\mathrm{n}}$ PREEMPTIVE: pre-emptive transplant.

${ }^{\mathrm{o}} \mathrm{RWT} 2$ : recipient weight.

Below, we analyze the importance of features in each cohort and show the influence of features over time using nonoverlapping cohorts.

\section{Feature Importance for Cohort 1}

According to the top features shown in Table 8, HLAMMs and the number of years on dialysis before transplant (VINTAGE) were the most important features with a relative importance of over $10 \%$. This observation has been confirmed in other studies [32,36]. Donor-recipient CMV status, donor-recipient race, end-stage renal disease diagnosis (ESRDDXSIMP), and functional status of the recipient were ranked as having medium importance with a relative score between $5 \%$ and $10 \%$. Donor-recipient race pairs and donor-recipient CMV pairs were noted to have more predictive influence in cohort 1 than in the other two cohorts.

\section{Feature Importance for Cohort 2}

Both HLAMMs and VINTAGE remained highly important in cohort 2. In addition, ESRDDXSIMP was noted as a highly important feature. Interestingly, we note that few features, such as donor age and recipient age, were rather insignificant in cohort 1 but were noted to be significant in both cohort 2 and further in cohort 3 .

\section{Feature Importance for Cohort 3}

ESRDDXSIMP showed a relative downward trend; however, it remained a highly significant feature. Unlike earlier cohorts, HLAMMs and VINTAGE were noted to not maintain their importance in the long term, whereas the recipient's status of diabetes was noted to be the most important feature, along with recipient age and their functional status. Donor age was noted to maintain a medium importance score between $5 \%$ and $10 \%$.

Figure 7 presents a heat map of the importance score to illustrate the changing influence of the top 25 features across the three cohorts. In addition to the top 10 features (Table 8), the heat map details the contribution of relatively less important features. It was interesting to see that few features (such as donor weight, recipient weight, and donor hypertension) had static importance 
across the three cohorts. This indicates that although these a certain value for the prediction models.

features were not deterministic for the cohorts, they possessed

Figure 7. Changing relevance of top 25 features over the three cohorts. AHD1: donor-recipient height difference; CIT: cold ischemia time; DAGE: donor age; DHT100: donor height; DHTN2: donor hypertension; DONCREAT: donor creatinine level; DRACESIMP: donor race; DRCMV: donor-recipient cytomegalovirus; DRRACE: donor-recipient race; DRWT: donor-recipient weight difference; DWT: donor weight; ECD: expanded criteria donor; ESRDDXSIMP: simplified end-stage renal disease diagnosis; FUNCTSTAT: functional status of the recipient; HLAMM: number of HLA mismatches; PKPRA: peak panel reactive antibody; PREEMPTIVE: preemptive transplant; RAGETX: recipient age; RCAD: recipient coronary artery disease; RDM2: recipient diabetes; RHT2100: recipient height; RHTN: recipient hypertension; RRACESIMP: recipient race; RWT2: recipient weight; VINTAGE: number of years on dialysis before transplantation.

\begin{tabular}{|c|c|c|c|}
\hline VINTAGE - & $12 \%$ & $11 \%$ & $2 \%$ \\
\hline RWT2- & $1 \%$ & $2 \%$ & $2 \%$ \\
\hline RRACESIMP - & $2 \%$ & $2 \%$ & $1 \%$ \\
\hline $\mathrm{RHTN}-$ & $2 \%$ & $1 \%$ & $0 \%$ \\
\hline RHT2100- & $1 \%$ & $2 \%$ & $1 \%$ \\
\hline RDM2 - & $3 \%$ & $2 \%$ & $16 \%$ \\
\hline $\mathrm{RCAD}-$ & $4 \%$ & $1 \%$ & $1 \%$ \\
\hline RAGETX - & $3 \%$ & $9 \%$ & $17 \%$ \\
\hline PREEMPTIVE - & $2 \%$ & $3 \%$ & $2 \%$ \\
\hline PKPRA - & $3 \%$ & $3 \%$ & $1 \%$ \\
\hline HLAMM - & $13 \%$ & $10 \%$ & $4 \%$ \\
\hline FUNCTSTAT - & $7 \%$ & $4 \%$ & $11 \%$ \\
\hline ESRDDXSIMP - & $9 \%$ & $18 \%$ & $13 \%$ \\
\hline ECD - & $0 \%$ & $1 \%$ & $3 \%$ \\
\hline DWT - & $1 \%$ & $1 \%$ & $1 \%$ \\
\hline DRWT - & $2 \%$ & $2 \%$ & $2 \%$ \\
\hline DRRACE - & $8 \%$ & $4 \%$ & $3 \%$ \\
\hline DRCMV - & $8 \%$ & $2 \%$ & $1 \%$ \\
\hline DRACESIMP - & $2 \%$ & $0 \%$ & $0 \%$ \\
\hline DONCREAT - & $0 \%$ & $1 \%$ & $1 \%$ \\
\hline DHTN2 - & $1 \%$ & $1 \%$ & $1 \%$ \\
\hline DHT100- & $1 \%$ & $1 \%$ & $1 \%$ \\
\hline DAGE - & $4 \%$ & $6 \%$ & $5 \%$ \\
\hline CIT - & $1 \%$ & $2 \%$ & $1 \%$ \\
\hline AHD1- & $2 \%$ & $3 \%$ & $2 \%$ \\
\hline
\end{tabular}

Analysis of the values of categorical features provided novel insights into the influence of a feature. Figure 8 presents a heat map of the importance of the value of the categorical features generated after transforming them into dummy features. 
Figure 8. Changing relevance of top 25 features (including dummy features) over the three cohorts. CIT: cold ischemia time; DAGE: donor age; DBMI: donor BMI; DHT100: donor height; DONCREAT: donor creatinine level; DRCMV_2: Donor positive recipient positive; DRRACE_1: Donor white recipient white; DWT: donor weight; ECD_0: Expanded criteria donor: no; ECD_1: Expanded criteria donor: yes; ESRDDXSIMP_2: End stage renal disease: diabetes mellitus; ESRDDXSIMP_3: End stage renal disease: polycystic kidney disease; ESRDDXSIMP_4: End stage renal disease: hypertension; FUNCTSTAT_1: Functional status of recipient: 100\% no complaints; HLAMM_5: Number of human leukocyte antigen mismatches: 5; PKPRA: peak panel reactive antibody; PREEMPTIVE_1: Preemptive transplant: yes; PREEMPTIVE_2: Preemptive transplant: no; RAGETX: recipient age; RBMI2: recipient BMI; RDM2_0: Recipient diabetes: no; RDM2_1: Recipient diabetes: yes; RHT2100: recipient height; RWT2: recipient weight; VINTAGE: number of years on dialysis before transplantation.

\begin{tabular}{|c|c|c|c|}
\hline VINTAGE - & $10 \%$ & $10 \%$ & $3 \%$ \\
\hline RWT2 - & $2 \%$ & $3 \%$ & $3 \%$ \\
\hline RHT2100- & $2 \%$ & $3 \%$ & $2 \%$ \\
\hline RDM2_1- & $2 \%$ & $2 \%$ & $8 \%$ \\
\hline RDM2_0-1 & $2 \%$ & $2 \%$ & $8 \%$ \\
\hline RBMI2 - & $2 \%$ & $3 \%$ & $3 \%$ \\
\hline RAGETX - & $3 \%$ & $7 \%$ & $12 \%$ \\
\hline PREEMPTIVE_2 - & $2 \%$ & $2 \%$ & $2 \%$ \\
\hline PREEMPTIVE_1 - & $2 \%$ & $2 \%$ & $2 \%$ \\
\hline PKPRA - & $2 \%$ & $2 \%$ & $2 \%$ \\
\hline HLAMM_5- & $5 \%$ & $2 \%$ & $0 \%$ \\
\hline FUNCTSTAT_1- & $2 \%$ & $1 \%$ & $3 \%$ \\
\hline ESRDDXSIMP_4 - & $0 \%$ & $1 \%$ & $1 \%$ \\
\hline ESRDDXSIMP_3- & $2 \%$ & $5 \%$ & $2 \%$ \\
\hline ESRDDXSIMP_2- & $5 \%$ & $5 \%$ & $6 \%$ \\
\hline ECD_1- & $1 \%$ & $1 \%$ & $2 \%$ \\
\hline ECD_0-1 & $1 \%$ & $1 \%$ & $2 \%$ \\
\hline DWT - & $2 \%$ & $2 \%$ & $2 \%$ \\
\hline DRRACE_1- & $3 \%$ & $1 \%$ & $0 \%$ \\
\hline DRCMV_2- & $6 \%$ & $1 \%$ & $0 \%$ \\
\hline DONCREAT - & $3 \%$ & $2 \%$ & $2 \%$ \\
\hline DHT100- & $3 \%$ & $2 \%$ & $2 \%$ \\
\hline $\mathrm{DBMI}-$ & $2 \%$ & $2 \%$ & $2 \%$ \\
\hline DAGE - & $4 \%$ & $6 \%$ & $5 \%$ \\
\hline \multirow[t]{2}{*}{$\mathrm{CIT}$} & $2 \%$ & $2 \%$ & $2 \%$ \\
\hline & & & \\
\hline
\end{tabular}




\section{Discussion}

\section{Principal Findings}

The cross-cohort prediction results (Table 6) confirm the efficacy of the classifiers-the prediction model for cohort 3 (ie, AdaBoost) correctly offers a high prediction score for data from cohort $1(72 \%)$ and cohort $2(75 \%)$. The prediction model for cohort 2 offers a high prediction score for cohort 1 data (79\%) but a low prediction score for cohort $3(58 \%)$ data. The classifier for cohort 1 (ie, SVM) gave low prediction scores for data from cohort $2(42 \%)$ and cohort $3(29 \%)$. Interestingly, the highest prediction score by a cohort-specific classifier was always achieved for data from its respective cohort. The prediction modeling results confirmed that the prediction models were highly sensitive to their respective cohorts.

\section{Comparing Prediction Performance With Prior Studies}

We compared the prediction performance of our ML-based prediction models with comparable organ transplant studies that involved similar-sized observations and temporal windows. Table 9 summarizes the findings of the two studies for each cohort. There have been several other studies $[19,31,32,35,37,38]$ to predict the short-term graft status of different organ transplants, but because of their small data set, these do not serve as a meaningful comparison.

Table 9. Prediction scores of similar studies.

\begin{tabular}{llllllll}
\hline Time & Study & Model & Size & Data set & Metric & Score (\%) & Our score (\%) \\
\hline 1 year & Lin et al [16] & ANN ${ }^{\mathrm{a}}$ and LR $^{\mathrm{b}}$ & 46,414 & UNOS $^{\mathrm{c}}$ & AUC $^{\mathrm{d}}$ & 73 & 82 \\
1 year & Dag et al [23] & LR & 15,580 & UNOS & AUC & 63 & 82 \\
5 years & Tiong et al [39] & Nomogram & 20,085 & UNOS & C-index & 71 & 69 \\
5 years & Lin et al [16] & ANN & 17,856 & UNOS & AUC & 77 & 69 \\
7 years & Lin et al [16] & ANN & 10,250 & UNOS & AUC & 82 & 81 \\
14 years & Luck et al [40] & ANN & 46,098 & SRTR & C-index & 65 & 81 \\
\hline
\end{tabular}

${ }^{\mathrm{a}} \mathrm{ANN}$ : artificial neural network.

${ }^{\mathrm{b}} \mathrm{LR}$ : logistic regression.

${ }^{\mathrm{c}}$ UNOS: United Network of Organ Sharing.

${ }^{\mathrm{d}} \mathrm{AUC}$ : area under the curve.

${ }^{\mathrm{e}} \mathrm{C}$-index: concordance index.

${ }^{\mathrm{f}}$ SRTR: Scientific Registry of Transplant Recipients.

When comparing our results with prior studies, it is noted that although our cohort 2 prediction performance (ie, graft status prediction over a 5-year period) is lower than that of Lin et al [16], it was based on a much smaller data set that included 10,641 survivals and 7215 failures, whereas we analyzed 23,475 failures and 29,352 survivals. Similarly, Tiong et al [39] analyzed a smaller sample of 20,085 living donor transplant recipients to achieve a concordance index of $71 \%$. Our cohort 3 prediction performance is marginally lower compared with Lin et al [16], who predicted a 7-year graft survival with an $82 \%$ AUC score, whereas our cohort 3 prediction model covers a much longer (17 years) temporal window and achieves a comparable prediction score. Using a similar number of transplants, Luck et al [40] achieved a much lower concordance index between $63 \%$ and $66 \%$ for 14 -years graft survival.

\section{Limitations and Future Work}

A limitation of our research lies in the removal of censored instances. We removed all successful cases that were censored before 8 years following transplant. Although this type of approach has previously been used, including censored cases is a potential consideration for future analyses.

\section{Conclusions}

Understanding the impact of donor and recipient factors that predict short- and long-term kidney transplant allograft survival is important for patients and providers. Kidney transplantation is the optimal form of kidney replacement therapy, but kidney allografts are a limited resource. In addition, the alternative to kidney transplantation (ie, dialysis) is considerably costlier.

In this study, we present an ML-based framework to predict the status of kidney allografts, based on donor-recipient features, over a period of 17 years. We applied ML-based data analysis methods for feature engineering to reduce data dimensionality, develop prediction models for three distinct temporal cohorts, and investigate the changing relevance of clinical features across different temporal cohorts. We introduced the concept of nonoverlapped cohorts to analyze the changing relevance of features in three defined periods. In conclusion, our results emphasize that ML can be effective in predicting graft survival using donor and recipient factors that are routinely collected as part of patient care. As a next step, we plan to incorporate the prediction models into clinical care at the time of allocation; models that best predict short- and long-term kidney graft survival may be used as a pragmatic prognostic tool to aid clinicians in maximizing the best possible matching of donors and recipients while preserving existing allocation rules that are used to promote equity [41]. 


\section{Acknowledgments}

The data reported here have been supplied by the Hennepin Healthcare Research Institute as the contractor for the SRTR. The interpretation and reporting of these data are the responsibility of the authors and in no way should be seen as an official policy of or interpretation by the SRTR or the US Government.

\section{Authors' Contributions}

SAAN and SSRA were responsible for the overall data analysis methodology, data analysis using ML algorithms, evaluation of the data analysis results, and writing of the manuscript. KT and AV provided clinical expertise in defining the problem, interpretation of the data, preprocessing of the data and interpretation of the results, providing the data from the data source, and editing the manuscript for clinical clarity and purpose. PCR facilitated the setting up and conducting of data analysis experiments. All authors critically reviewed the manuscript for scientific content and approved the final manuscript for publication.

\section{Conflicts of Interest}

None declared.

\section{Multimedia Appendix 1}

Supplementary tables.

[DOC File, 24 KB-Multimedia Appendix 1]

\section{References}

1. Sullivan CE. Kidney Transplantation. Transplantation 1968;6(5):753. [doi: 10.1097/00007890-196808000-00031]

2. Hall YN, Chertow GM. End stage renal disease. BMJ Clin Evid 2007 Oct 17;2007:1048-1059 [FREE Full text] [Medline: $\underline{19450356]}$

3. Go AS, Chertow GM, Fan D, McCulloch CE, Hsu C. Chronic Kidney Disease and the Risks of Death, Cardiovascular Events, and Hospitalization. N Engl J Med 2004 Sep 23;351(13):1296-1305. [doi: 10.1056/nejmoa041031]

4. Rashidi Khazaee P, Bagherzadeh J, Niazkhani Z, Pirnejad H. A dynamic model for predicting graft function in kidney recipients' upcoming follow up visits: A clinical application of artificial neural network. Int J Med Inform 2018 Nov;119:125-133 [FREE Full text] [doi: 10.1016/j.ijmedinf.2018.09.012] [Medline: 30342680]

5. Schold J, Segev DL. Increasing the pool of deceased donor organs for kidney transplantation. Nat Rev Nephrol 2012 Mar 27;8(6):325-331 [FREE Full text] [doi: 10.1038/nrneph.2012.60] [Medline: 22450438]

6. Conservative Care - The Kidney Foundation of Canada | La Fondation canadienne du rein. URL: https://www.kidney.ca/ conservative-care [accessed 2019-11-15]

7. About Chronic Kidney Disease | National Kidney Foundation. URL: https://www.kidney.org/atoz/content/ about-chronic-kidney-disease [accessed 2019-11-15]

8. Perl J. Kidney transplant failure: failing kidneys, failing care? Clin J Am Soc Nephrol 2014 Jul;9(7):1153-1155 [FREE Full text] [doi: 10.2215/CJN.04670514] [Medline: 24903388]

9. Vinson A, Kiberd B, Davis R, Tennankore K. Nonimmunologic Donor-Recipient Pairing, HLA Matching, and Graft Loss in Deceased Donor Kidney Transplantation. Transplantation Direct 2019 Jan;5(1):e414 [FREE Full text] [doi: 10.1097/txd.0000000000000856]

10. Rao P, Schaubel D, Guidinger MK, Andreoni KA, Wolfe RA, Merion RM, et al. A comprehensive risk quantification score for deceased donor kidneys: the kidney donor risk index. Transplantation 2009 Jul 27;88(2):231-236. [doi: 10.1097/TP.0b013e3181ac620b] [Medline: 19623019]

11. Young A, Knoll GA, McArthur E, Dixon SN, Garg AX, Lok CE, et al. Is the Kidney Donor Risk Index a Useful Tool in Non-US Patients? Can J Kidney Health Dis 2018;5:2054358118791148 [FREE Full text] [doi: 10.1177/2054358118791148] [Medline: 30083367]

12. Senanayake S, White N, Graves N, Healy H, Baboolal K, Kularatna S. Machine learning in predicting graft failure following kidney transplantation: A systematic review of published predictive models. Int J Med Inform 2019 Oct;130:103957 [FREE Full text] [doi: 10.1016/j.ijmedinf.2019.103957] [Medline: 31472443]

13. Rao V, Behara R, Agrawal A. Predictive modeling for organ transplantation outcomes. 2014 Nov 10 Presented at: Proc IEEE 14th Int Conf Bioinforma Bioeng BIBE 405?408; 2014; Boca Raton, FL, USA URL: https://ieeexplore.ieee.org/ document/7033613/ [doi: 10.1109/BIBE.2014.58]

14. Brown T, Elster E, Stevens K, Graybill JC, Gillern S, Phinney S, et al. Bayesian modeling of pretransplant variables accurately predicts kidney graft survival. Am J Nephrol 2012;36(6):561-569 [FREE Full text] [doi: 10.1159/000345552] [Medline: 23221105]

15. Topuz K, Zengul F, Dag A, Almehmi A, Yildirim M. Predicting graft survival among kidney transplant recipients: A Bayesian decision support model. Decision Support Systems 2018 Feb;106:97-109 [FREE Full text] [doi:

10.1016/j.dss.2017.12.004] 
16. Lin R, Horn S, Hurdle J, Goldfarb-Rumyantzev AS. Single and multiple time-point prediction models in kidney transplant outcomes. J Biomed Inform 2008 Dec;41(6):944-952 [FREE Full text] [doi: 10.1016/j.jbi.2008.03.005] [Medline: 18442951]

17. Axelrod D, Schnitzler M, Xiao H, Irish W, Tuttle-Newhall E, Chang SH, et al. An economic assessment of contemporary kidney transplant practice. Am J Transplant 2018 May;18(5):1168-1176 [FREE Full text] [doi: 10.1111/ajt.14702] [Medline: $\underline{29451350]}$

18. Lee D, Kanellis J, Mulley WR. Allocation of deceased donor kidneys: A review of international practices. Nephrology (Carlton) 2019 Jun;24(6):591-598 [FREE Full text] [doi: 10.1111/nep.13548] [Medline: 30536674]

19. George B, Seals S, Aban I. Survival analysis and regression models. J Nucl Cardiol 2014 Aug;21(4):686-694 [FREE Full text] [doi: 10.1007/s12350-014-9908-2] [Medline: 24810431]

20. Li J, Serpen G, Selman S. Bayes net classifiers for prediction of renal graft status and survival period. World Academy of Science, Engineering and Technology, Open Science Index 39, International Journal of Biomedical and Biological Engineering 2010:88-94. [doi: 10.5281/zenodo.1334844]

21. Mamoshina P, Vieira A, Putin E, Zhavoronkov A. Applications of Deep Learning in Biomedicine. Mol Pharm 2016 May 02;13(5):1445-1454 [FREE Full text] [doi: 10.1021/acs.molpharmaceut.5b00982] [Medline: 27007977]

22. Sadati N, Zafar M, Babu R. Representation Learning with Autoencoders for Electronic Health Records: A Comparative Study. Computing Research Repository. URL: https://arxiv.org/abs/1908.09174 [accessed 2020-11-20]

23. Dag A, Oztekin A, Yucel A, Bulur S, Megahed F. Predicting heart transplantation outcomes through data analytics. Decision Support Systems 2017 Feb;94:42-52 [FREE Full text] [doi: 10.1016/j.dss.2016.10.005]

24. Yoon J, Zame W, Banerjee A, Cadeiras M, Alaa AM, van der Schaar M. Personalized survival predictions via Trees of Predictors: An application to cardiac transplantation. PLoS One 2018;13(3):e0194985 [FREE Full text] [doi: 10.1371/journal.pone.0194985] [Medline: 29590219]

25. Van Belle V, Pelckmans K, Van Huffel S, Suykens JAK. Support vector methods for survival analysis: a comparison between ranking and regression approaches. Artif Intell Med 2011 Oct;53(2):107-118 [FREE Full text] [doi: 10.1016/j.artmed.2011.06.006] [Medline: 21821401]

26. Dorado-Moreno M, Pérez-Ortiz M, Gutiérrez PA, Ciria R, Briceño J, Hervás-Martínez C. Dynamically weighted evolutionary ordinal neural network for solving an imbalanced liver transplantation problem. Artif Intell Med 2017 Mar;77:1-11. [doi: 10.1016/j.artmed.2017.02.004] [Medline: 28545607]

27. Hong K, Iribarne A, Worku B, Takayama H, Gelijns AC, Naka Y, et al. Who is the high-risk recipient? Predicting mortality after heart transplant using pretransplant donor and recipient risk factors. Ann Thorac Surg 2011 Aug;92(2):520-7; discussion 527 [FREE Full text] [doi: 10.1016/j.athoracsur.2011.02.086] [Medline: 21683337]

28. Lofaro D, Maestripieri S, Greco R, Papalia T, Mancuso D, Conforti D, et al. Prediction of chronic allograft nephropathy using classification trees. Transplant Proc 2010 May;42(4):1130-1133 [FREE Full text] [doi: 10.1016/j.transproceed.2010.03.062] [Medline: 20534242]

29. Medved D, Nugues P, Nilsson J. Predicting the outcome for patients in a heart transplantation queue using deep learning. Annu Int Conf IEEE Eng Med Biol Soc 2017 Jul;2017:74-77. [doi: 10.1109/EMBC.2017.8036766] [Medline: 29059814]

30. Chawla NV, Bowyer KW, Hall LO, Kegelmeyer WP. SMOTE: Synthetic Minority Over-sampling Technique. jair 2002 Jun 01;16:321-357 [FREE Full text] [doi: 10.1613/jair.953]

31. Varoquaux G, Buitinck L, Louppe G, Grisel O, Pedregosa F, Mueller A. Scikit-learn. GetMobile: Mobile Comp. and Comm 2015 Jun;19(1):29-33 [FREE Full text] [doi: 10.1145/2786984.2786995]

32. Chollet F. Keras. URL: https://github.com/fchollet/keras [accessed 2020-09-15]

33. Medved D, Nugues P, Nilsson J. Selection of an optimal feature set to predict heart transplantation outcomes. Annu Int Conf IEEE Eng Med Biol Soc 2016 Aug;2016:3290-3293 [FREE Full text] [doi: 10.1109/EMBC.2016.7591431] [Medline: 28269008]

34. Thongkam J, Xu G, Zhang Y. AdaBoost algorithm with random forests for predicting breast cancer survivability. 2008 Presented at: IEEE International Joint Conference on Neural Networks; 1-8 June 2008; Hong Kong, China URL: https:/ /doi.org/10.1109/IJCNN.2008.4634231 [doi: 10.1109/IJCNN.2008.4634231]

35. Tennankore K, Kim S, Alwayn I, Kiberd BA. Prolonged warm ischemia time is associated with graft failure and mortality after kidney transplantation. Kidney Int 2016 Mar;89(3):648-658 [FREE Full text] [doi: 10.1016/j.kint.2015.09.002] [Medline: 26880458]

36. Piros P, Ferenci T, Fleiner R, Andréka P, Fujita H, Főző L, et al. Comparing machine learning and regression models for mortality prediction based on the Hungarian Myocardial Infarction Registry. Knowledge-Based Systems 2019 Sep;179:1-7 [FREE Full text] [doi: 10.1016/j.knosys.2019.04.027]

37. Adegoke V, Chen D, Banissi E, Barikzai S. Prediction of breast cancer survivability using ensemble algorithms. 2017 Oct 18 Presented at: International Conference on Smart Systems and Technologies (SST); 2017; Osijek, Croatia URL: https:/ /doi.org/10.1109/SST.2017.8188699 [doi: 10.1109/SST.2017.8188699]

38. Yoo K, Noh J, Lee H, Kim DK, Lim CS, Kim YH, et al. A Machine Learning Approach Using Survival Statistics to Predict Graft Survival in Kidney Transplant Recipients: A Multicenter Cohort Study. Sci Rep 2017 Aug 21;7(1):8904 [FREE Full text] [doi: 10.1038/s41598-017-08008-8] [Medline: 28827646] 
39. Tiong H, Goldfarb D, Kattan MW, Alster JM, Thuita L, Yu C, et al. Nomograms for predicting graft function and survival in living donor kidney transplantation based on the UNOS Registry. J Urol 2009 Mar;181(3):1248-1255 [FREE Full text] [doi: 10.1016/j.juro.2008.10.164] [Medline: 19167732]

40. Luck M, Sylvain T, Cardinal H. Deep Learning for Patient-Specific Kidney Graft Survival Analysis. ArXiv. 2017. URL: https://arxiv.org/abs/1705.10245 [accessed 2020-10-24]

41. Loupy A, Aubert O, Orandi BJ, Naesens M, Bouatou Y, Raynaud M, et al. Prediction system for risk of allograft loss in patients receiving kidney transplants: international derivation and validation study. BMJ 2019 Sep 17;366:14923 [FREE

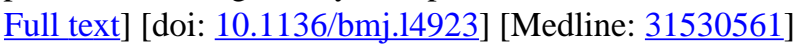

\author{
Abbreviations \\ AdaBoost: adaptive boosting \\ ANN: artificial neural network \\ AUC: area under the curve \\ CMV: cytomegalovirus \\ ESRDDXSIMP: end-stage renal disease diagnosis \\ LR: logistic regression \\ ML: machine learning \\ RF: random forest \\ SMOTE: Synthetic Minority Oversampling Technique \\ SRTR: Scientific Registry of Transplant Recipients \\ SVM: support vector machine \\ VINTAGE: number of years on dialysis before transplant
}

\author{
Edited by R Kukafka; submitted 04.01.21; peer-reviewed by P Giabbanelli, E Mahmoudi; comments to author 01.02.21; revised \\ version received 10.03.21; accepted 06.05.21; published 27.08.21 \\ Please cite as: \\ Naqvi SAA, Tennankore K, Vinson A, Roy PC, Abidi SSR \\ Predicting Kidney Graft Survival Using Machine Learning Methods: Prediction Model Development and Feature Significance Analysis \\ Study \\ J Med Internet Res 2021;23(8):e26843 \\ URL: https://www.jmir.org/2021/8/e26843 \\ doi: $10.2196 / 26843$ \\ PMID:
}

(C) Syed Asil Ali Naqvi, Karthik Tennankore, Amanda Vinson, Patrice C Roy, Syed Sibte Raza Abidi. Originally published in the Journal of Medical Internet Research (https://www.jmir.org), 27.08.2021. This is an open-access article distributed under the terms of the Creative Commons Attribution License (https://creativecommons.org/licenses/by/4.0/), which permits unrestricted use, distribution, and reproduction in any medium, provided the original work, first published in the Journal of Medical Internet Research, is properly cited. The complete bibliographic information, a link to the original publication on https://www.jmir.org/, as well as this copyright and license information must be included. 